\title{
К ВОПРОСУ ОБ ОНТОЛОГИЧЕСКОМ СТАТУСЕ ОБЪЕКТА «ТО, БОЛЬШЕ ЧЕГО НЕЛЬЗЯ ПРЕДСТАВИТЬ ${ }^{1}$
}

\author{
В.В. Горбатов
}

\section{1 Проблемы интерпретации «единственного аргумента»}

В трактате «Прослогион» Ансельм Кентерберийский предложил простое и изящное доказательство бытия Бога, одна из наиболее популярных формулировок которого (из главы II) гласит:

Если то, больше чего нельзя ничего себе представить, существует только в уме, тогда то, больше чего нельзя себе представить, есть то, больше чего можно представить себе. Но этого, конечно, не может быть. Итак, без сомнения, нечто, большее чего нельзя себе представить, существует и в уме, и в действительности [2].

Несмотря на то, что в литературе идеи «Прослогиона» часто сближаются и даже отождествляются с «онтологическим аргументом», существует множество причин, по которым этого делать не следует. Важнее всего то, сама постановка главной задачи в «единственном аргументе» серьезно отличается от того, как она изложена у Канта. Речь в нем идет отнюдь не о выведении объективного существования вещи из ее понятия, а об обосновании возможности перехода от интенционального объекта к реальному, от существования в уме (необязательно в форме понятия) к существованию в действительности (см.: [4, с. 135]).

\footnotetext{
${ }^{1}$ Исследование осуществлено в рамках Программы «Научный фонд НИУ ВШЭ» в 2013-2014 гг., проект № 12-01-0111.
} 
Остановимся на этом моменте. Дело не в том, что субъект сначала имеет в уме врожденную или приобретенную идею Бога, понятие всесовершенного существа, а потом уже неким образом аналитически выводит из данного понятия действительное существование соответствующего объекта (в широком смысле слова). Напротив, Ансельм описывает ситуацию, в которой субъект, минуя стадию образования понятия, самой попыткой понимания (при условии, что эта попытка серьезна и искренна) порождает в своем сознании особый интенциональный объект - «то, больше чего нельзя представить» $($ iqmcn $),-$ после чего с помощью нехитрых логических процедур «переоткрывает» этот же объект для себя как реальный. Стоит напомнить, что понятием о Боге, по Ансельму, строго говоря, обладать вообще невозможно - ведь iqmcn всегда превосходит то, что лишь представляется нами как iqmcn. В главе XV «Прослогиона» читаем: «Господи, Ты не только то, больше чего нельзя представить, но сам ты есть нечто большее, чем можно представитъ» (курсив мой- В.Г.) [2, с. 138].

Но тогда возникает принципиальный вопрос: как же возможно такое вне-понятийное полагание объекта? Полагание, в котором мысль отказывается от своих притязаний на схватывание сущности мыслимого, но, в то же время, отчетливо распознает ускользающее от нее как особый объект и крепко удерживает референцию к нему? Если внимательно читать «Прослогион», ответ напрашивается сам собой: понятию помогает слово как особый медиум отношения сознания к реальности, причастный самой реальности. Как подчеркивает А.В. Басос,

\begin{abstract}
важно понимать, что ансельмово доказательство бытия Бога не предполагает предварительного введения понятия (в современном смысле) высшей сущности. «То, больше чего нельзя представить» - это не понятие Бога, а сам Бог, усматриваемый во внутреннем речении. Речь, слово - это внутренняя сущность любой вещи, поскольку в основу мира положено Слово [3, с. 104].
\end{abstract}

Таким образом, вся суть аргументации Ансельма заключается в том, что несуществование iqmcn запрещено устройством нашего языка. По крайней мере в одном случае - случае с Богом - внутренняя принадлежность изрекаемого слова к сфере, где бытийствует мышление и мыслится бытие, не позволяет мышлению и бытию распасться на два отдельных мира: «само произнесение слова „Бог“ (разумеется, с сопутствующим пониманием) влечет за собой необходимость Его существования» [3, с. 104].

Следует подчеркнуть, что данное доказательство черпает свои силы не столько в чистой логике или эпистемологии, сколько в теории 
значения. На первом отрезке рассуждения Ансельму важно заставить оппонента мысленно закрепить сам объект спора - зафиксировать то, о чем именно «безумец» утверждает, что его нет. Исходя из принципа «все, что понимается, есть в понимании» (quidquid intelligitur in intellectu est), автор настойчиво требует от неверующего если не сформировать понятие о «том, больше чего нельзя представить» (как мы помним, это в данном случае невозможно), то хотя бы отчетливо представить, как организована референция подобного выражения. А затем ему остается просто сделать соответствующие выводы.

Неслучайно для иллюстрации различия между существованием в уме (in intellectu) и в действительности (in re) Ансельм проводит аналогию с художественным замыслом:

Так, когда художник заранее обдумывает то, что будет делать, он, правда, имеет в уме то, чего еще не сделал, но отнюдь не подразумевает его существования. А когда он уже нарисовал, он и имеет в уме, и мыслит как существующее то, что уже сделал [2, с. 128].

Для нас важно, что ситуация художника в этом смысле сильно отличается от ситуации математика, занимающегося вычислениями или продумывающего доказательство теоремы. Ведь художник в своем уме имеет саму картину, а не отвлеченное рациональное понятие о ней. А.В. Басос интерпретирует данный пассаж таким образом:

Еще даже не воплотив свой замысел в жизнь, художник пока только проговаривает свою картину с помощью некоего внутреннего речения (locutio mentis). Это «речение» имеет место, когда сама вещь созерцается взором мысли в уме. Т. е. здесь нет никакого разделения на предмет и понятие предмета в уме. Есть, собственно, всегда только сама вещь, и «речение» вещи - это созерцание ее как таковой [3, с. 156].

Однако вывод А.В. Басоса о том, что «с доказательством Ансельма невозможно согласиться, не приняв предварительно тезис о творении мира по Слову» [3, с. 165$]$, представляется слишком сильным и односторонним. На мой взгляд, необходимо не столько «вызволение Ансельма из контекста кантовской философии и утверждение его в контексте проблем философии Средневековья» [3, с. 166], сколько переосмысление его с учетом достижений современной аналитической философии. Есть основания считать, что заложенный в unum argumentum взгляд на язык и его отношение к бытию не сводится к христианской догматике; он может быть отделен от сугубо теологических воззрений автора и подвергнут экспликации в терминах современных логико-семантических концепций. 


\section{2 Ансельмов аргумент как перформативное доказательство}

Большим шагом в этом направлении является предложенная Е.Г. Драгалиной-Черной интерпретация «единственного аргумента» как перформативного доказательства $[4,5,6]$.

$\mathrm{C}$ перформативной точки зрения, любое доказательство есть демонстрация того, каким образом исполнение простых когнитивных актов делает возможным компетентное исполнение более сложного когнитивного акта и оказывается переходом не от одних истинных высказываний к другим, а от одних обоснованных действий к другим, получающим, таким образом, свою обоснованность [6, с. 138].

Например, картезианское cogito ergo sum явно относится к такому классу доказательств: в нем не предикат «быть мыслящим», но сам акт мышления удостоверяет акт существования мыслящего субъекта, причем существования именно в качестве «мыслящей вещи» (res cogitans) и только до тех пор, пока продолжается само мышление.

В случае единственного аргумента, такого рода обосновывающим действием оказывается референция к «тому, больше чего нельзя представить». Референция к столь необычному объекту представляет собой особый рефлексивный акт, в котором «познающий интеллект осознает себя познающим», будучи обращен сразу к двум уровням: предметному уровню «вещи, о которой идет речь», и метауровню «мысли об этой вещи» $[6$, с. 138].

Другими словами, суть ансельмова доказательства сводится к демонстрации того, что, «осуществляя рефлексивную референцию к „тому, больше чего нельзя представить“, рациональный субъект не может мыслить объект своей референции несуществующим» [6, с. 139].

Обсуждая идею перформативного доказательства, необходимо отметить несколько моментов. Во-первых, его успешность напрямую зависит от действительного, а не притворного осуществления субъектом соответствующих когнитивных (и речевых) актов. Во-вторых, предполагается, что осуществляющий эти акты субъект обладает способностью к рефлексии. В-третьих, рефлексия снабжает субъекта как бы «двойной оптикой»: помимо обычной перспективы «от третьего лица» он приобретает также перспективу «от первого лица».

Таким образом, свои собственные акты и их результаты субъект может теперь рассматривать не только «снаружи», то есть в предметном плане («Декарт мыслит»), но и «изнутри», то есть в плане феноменальном («Декарт - это на самом деле я, а его/мое мышление происходит здесь и сейчас»). Совершить перформативное доказательство 
за кого-то другого («Декарт мыслит, следовательно, он существует») невозможно.

Каков же логический статус перформативных доказательств? Следует ли их отнести к дедуктивным рассуждениям, или они дают нам всего лишь вероятностное, индуктивное знание? На эти вопросы трудно дать однозначный ответ. В первом приближении можно лишь констатировать, что результатом перформативного доказательства должно являться достижение определенной очевидности, хотя природа этой очевидности тесно связана с перспективой «от первого лица» и сильно отличается от очевидности таких аналитических утверждений как « $1+1=2 »$ или «квадрат гипотенузы равен сумме квадратов катетов». Само исполнение когнитивных актов, в строгом смысле слова, никому ничего не доказывает - кроме самого субъекта, да и то при условии соблюдения перечисленных выше ограничений. Оно, скорее, организует некое пространство очевидности, привилегированный доступ к которому есть лишь у того, кто эти акты совершает, а у всех остальных только в меру их способности поставить себя на его место.

Именно так, на мой взгляд, устроено рассуждение Ансельма. Он озабочен не тем, как достичь абстрактной (предметной) очевидности «от третьего лица», а тем, как отвести «безумца» в некое «умное место», откуда ему станет видно то, что видно самому Ансельму. Поэтому, как справедливо замечает С.С. Аверинцев, здесь речь идет не о «доказывании», но о «показывании» бытия Бога ${ }^{2}[1$, с. 34]. Дейктическая функция «внутреннего речения» о «том, больше чего нельзя ничего представить» явно важнее описательной, именно она служит ключом к пониманию конструкции всего аргумента.

Ансельмово «то, больше чего нельзя ничего представить»- не столько дескрипция, сколько дейктическое выражение (наподобие слов «то», «это», «здесь», «сейчас» и пр.). А это значит, что его референция организована не так, как референция обычных описательных имен. В частности, она оказывается чувствительной к контексту произнесения и обладает способностью «сдвигаться» при его изменении ${ }^{3}$. Однако подобная зависимость от обстоятельств употребления

\footnotetext{
${ }^{2}$ Различение этих терминов идет от Аристотеля, и применительно к вопросам богословия еще Климент Александрийский пояснял, что, поскольку доказательство есть логическое выведение вещи из более глубоких и более первичных начал, к безначальному Началу всех начал оно принципиально неприменимо (Clementis Alexandrini Stromata V, 82): «Никакое знание его [Бога] не может быть доказательным (курсив мой - В.Г.), поскольку любое такое знание должно базироваться на первых и уже известных принципах. Но ничто не предшествует не рожденному».

${ }^{3}$ Р.О. Якобсон называл такие элементы языка «шифтерами».
} 
не приводит к референциальному хаосу, поскольку сам механизм «сдвига» референции зафиксирован довольно четко и гарантирует некоторую регулярность. Зная, как именно изменяются обстоятельства произнесения дейктического выражения, можно всегда вычислить его денотат.

\section{3 «Смешанный» характер тезиса о существовании $I Q M C N$}

Д. Каплан [10] называл ту часть значения указательных (индексных) выражений, которая, несмотря на варьирование контекста, обеспечивает подобную регулярность, характером (character) - в противоположность содержанию (content), которое как раз подвержено изменениям. Понимая характер дейктического выражения, мы вовсе не обязаны знать его содержание (понятие, интенсионал), и наоборот. Если содержание является ответом на вопрос «что означает данное выражение?», то характер можно считать ответом на более фундаментальный вопрос: «как организована его референция?».

Я считаю, что предложенная Капланом модель очень удачно репрезентирует базовые интуиции Ансельма относительно вне-понятийного способа фиксации значения. И это влечет необходимость пересмотра логического статуса обосновываемого средневековым схоластом тезиса. Вопреки расхожим толкованиям, заключение «единственного аргумента» вовсе не претендует на метафизическую необходимость существования iqmcn - оно скорее утверждает априорную, связанную с языком (в том смысле, в котором принятие некоего языка мышления предшествует осуществлению самих мыслительных актов) очевидность Его существования.

Путь, предлагаемый в «Прослогионе», лежит не от понимания содержания лингвистической конструкции «то, больше чего нельзя представить» (iqmcn) через прослеживание этого iqmcn сквозь все возможные миры к констатации его необходимого существования («каким бы ни был мир, iqmcn в нем существует»). На самом деле, этот путь начинается с понимания характера рассматриваемого выражения, а далее ведет нас через схватывание заложенной в нем регулярности («каким бы ни было мое место в мире, мой контекст употребления „iqmcn“, наличие денотата этого выражения a priori обеспечивается его характером») к утверждению о немыслимости несуществования iqmcn. Другими словами, несмотря на «плаваю- 
щий» характер референции «iqmcn», из-за которого существование соответствующего объекта не может быть детерминировано метафизически, оно все-таки детерминировано эпистемически - гарантировано устройством моего языка, за пределы которого я не могу выйти, не приостановив свои когнитивные и речевые акты.

На первый взгляд, существование истин, которые были бы априорными (в указанном смысле), но при этом не выражали метафизической необходимости (или наоборот - были бы необходимыми, но не выражали априорного знания), может показаться сомнительным ${ }^{4}$. Но на самом деле, после открытия Саулом Крипке связи между именованием и необходимостью [7] представление о подобных «смешанных истинах» прочно утвердилось в аналитической философии языка и эпистемологии. Так, согласно Крипке, существует множество утверждений, которые познаваемы только эмпирически, но в то же время истинны во всех возможных мирах. Хрестоматийный пример-утверждение «Геспер = Фосфор». Познание этой истины возможно только из опыта, но поскольку «Геспер» и «Фосфор» являются жесткими десигнаторами, во всех мирах они должны обозначать одно и то жеа именно, Венеру. Эпистемически возможно, считает Крипке, чтобы Геспер не был Фосфором, метафизически - нет.

С другой стороны, существует также множество утверждений, истинность которых очевидна априори, но метафизической необходимости не влечет. Возьмем предложение «Я здесь сейчас»-кто бы его не произносил, ни в одном возможном мире и ни в один момент времени оно не потребует эмпирической проверки со стороны произносящего. Ведь сама ситуация употребления этого предложения организована так, что денотаты выражениям «Я», «здесь» и «сейчас» всегда приписываются согласованно: произносящий (кем бы он ни оказался) обязательно в момент произнесения находится в месте произнесения. Однако если мы рассмотрим любое конкретное употребление данного предложения и установим соответствующе ему положение дел, то обнаружим, что это положение дел почти всегда оказывается контингентным. Так, пока ваш покорный слуга в данный момент пишет предложение «Я здесь сейчас», оно, исходя из контекста употребления, получает вполне определенный денотатположение дел Виктор Горбатов 27 мая 2013 г. в 19:00 находится в Москве. А это положение дел совсем не претендует на метафизи-

\footnotetext{
${ }^{4}$ Как известно, Кант не признавал такой возможности и строго отождествлял необходимость с априорностью. О преодолении этой кантианской догмы см.: [8].
} 
ческую необходимость (не является истинным во всех возможных мирах).

Конечно, очевидность утверждения «я здесь сейчас» несколько отличается от очевидности утверждения «то, больше чего нельзя представить, существует». Как минимум, последнее требует уточнения понятия «больше», и ниже этот вопрос еще будет поднят. Но тем не менее, важно подчеркнуть, что оба они (1) относятся к классу контингентных априорных истин и (2) сама процедура их верификации носит перформативный характер.

\section{4 Двумерная семантика и логика актуальности}

Двумерная семантика возникла еще в начале 80-х годов XX века на стыке эпистемологии и теории значения, но, из-за ограниченности рассматриваемых ею тем, долгое время оставалась маргинальным направлением логико-философского анализа. Ее пионеры были всецело поглощены анализом отдельных, весьма специфических категорий языковых выражений (индексикалы у Д. Каплана, описательные имена у Г. Эванса, выражения с оператором «актуально» у М. Дэвиса и Л. Хамберстоуна). Обобщенную версию двумерной семантики в конце 90-х предложили Д. Чалмерс и Ф. Джексон. В настоящее время двумерный подход, в связи с обнаружением большого количества интересных философских приложений, переживает новый всплеск популярности. Его теоретический аппарат широко используется в связи с дискуссиями о роли концептуального анализа в философии, о соотношении понятий мыслимости и (метафизической) возможности, о редукционизме и априоризме. Но наиболее удачным приложением семантики подобного рода является объяснение природы интересующих нас «смешанных истин».

В рамках двумерной семантики выделяются два измерения значения - первое измерение соответствует референту (экстенсионалу) рассматриваемого выражения, второе измерение отражает то, как в произвольно выбранном возможном мире референция рассматриваемого выражения зависит от различных внешних факторов (например, от самой структуры этого мира). Р. Столнейкер указывает, что

нам нужны два измерения, поскольку мы начинаем с того факта, что истинностное значение пропозиций (по крайней мере, контингентных пропозиций) зависит от фактов. Но поскольку идентификация пропозиции, выражаемой при помощи используемого нами предложения [utterance], также зависит от фактов, истинностное значение этого 
предложения будет зависеть от фактов двумя различными способами (курсив мой- В.Г.): во-первых, факты определяют, что именно сказано; во-вторых, они определяют, является ли сказанное истинным [11, с. 302].

Технически это достигается следующим образом. В отличие от карнаповской семантики, где экстенсионалы выражений варьируются по мирам, а интенсионалы остаются стабильными, двумерный подход допускает также варьирование интенсионалов. В зависимости от обстоятельств употребления (контекстов, эпистемических перспектив) одному и тому же выражению могут приписываться различные интенсионалы ${ }^{5}$. По сути, понятие интенсионала при этом расслаивается на несколько взаимосвязанных понятий.

Традиционно интенсионалы представляются как функции из множества возможных миров в множество экстенсионалов. Но при двумерном подходе миры играют сразу две роли-точек референции (которые детерминируют, что именно было сказано) и точек соотнесения (которые определяют истинностное значение сказанного). Для краткости иногда точки соотнесения называют $C$-мирами (от «counterfactual»-миры, рассмотренные в качестве контрфактических), а точки референции - $A$-мирами (от «actual»-миры, рассмотренные в качестве актуальных). Идея заключается в том, что рассматривая миры в качестве контрфактических, мы удерживаем свою эпистемическую перспективу неизменной, а сослагаем лишь фактическую сторону вопроса (например, представляем мир, в котором снег не был бы белым). Рассматривая же миры в качестве актуальных, мы сослагаем как раз свою эпистемическую перспективу (например, представляем мир, в котором слово «снег» не означало бы снег), оставляя фактическую сторону без изменения.

В связи с этим принято различать первичные и вторичные интенсионалы ${ }^{6}$. Первичные (или $A$-интенсионалы) представляют собой функции из множества $A$-миров (точек референции) в множество экстенсионалов; вторичные (или $C$-интенсионалы) - из множества $C$-миров (точек соотнесения) в множество экстенсионалов. Часто рассматриваются также двумерные интенсионалы (функции из множества пар

\footnotetext{
${ }^{5}$ Суть заключается в том, что даже жесткие десигнаторы, такие как «Геспер» и «Фосфор», несмотря на то, что во всех мирах они имеют один и тот же денотат (Венеру), могут все же иметь различное когнитивное значение. Это объясняет, почему утверждение «Геспер = Фосфор» является необходимым, но не априорным.

${ }^{6}$ В терминологии Д. Чалмерса. Идущее далее изложение двумерного подхода, в целом, соответствует статье Чалмерса [8], с небольшими изменениями.
} 
миров $^{7}$ в множество экстенсионалов) и диагональные интенсионалы (функции из множества пар миров, в которых первый и второй элементы совпадают, в множество экстенсионалов).

Например, если речь идет о повествовательных предложениях, то их двумерный интенсионал - функция из множества пар миров в множество истинностных значений:

$$
\operatorname{Int}_{2 D}(\varphi)_{w, v} \in\{1,0\}
$$

Первичным интенсионалом предложения в $C$-мире $w$ является множество $A$-миров, относительно которых оно истинно:

$$
\operatorname{Int}_{A}(\varphi)_{w}=\left\{v: \operatorname{Int}_{2 D}(\varphi)_{w, v}=1\right\}
$$

Вторичным интенсионалом предложения в $A$-мире $v$ является множество $C$-миров, относительно которых оно истинно:

$$
\operatorname{Int}_{C}(\varphi)_{v}=\left\{w: \operatorname{Int}_{2 D}(\varphi)_{w, v}=1\right\}
$$

Диагональным интенсионалом предложения служит множество таких миров, которые, выступая одновременно в двух ролях (в качестве $A$ - и $C$-мира), делают данное предложение истинным:

$$
\operatorname{Int}_{D}(\varphi)=\left\{w: \operatorname{Int}_{2 D}(\varphi)_{w, w}=1\right\}
$$

На все эти виды интенсионалов распространяется принцип композициональности: когда экстенсионал сложного выражения композиционально зависит от экстенсионалов его частей, каждый из его интенсионалов относительно того или иного индекса сопоставления ( $A$-мир, $C$-мир или их упорядоченная пара) зависит точно таким же композициональным образом от соответствующих интенсионалов его частей относительно данного индекса сопоставления.

Из всех введенных выше понятий для нас важнее всего понятие диагонального интенсионала, являющееся аналогом каплановского «характера». В нем заложена способность рассмотреть любой произвольно взятый мир как актуальный. Синтаксически эта процедура чаще всего выражается при помощи оператора актуальности « $A$ ». Этот оператор, независимо от его места в формуле, «разрывает» область действия любых уже имеющихся в ней модальных операторов и осуществляет соотнесение связанного им предложения к реальному миру. Рассмотрим классический пример, фигурирующий в статье Дэвиса и Хамберстоуна [9].

\footnotetext{
7 Эти пары являются упорядоченными и имеют вид < точка референции; точка соотнесения $>$.
} 


\section{Пусть дано предложение}

(1) Все красное могло бы быть блестящим.

Существуют по крайней мере три естественных интерпретации этого предложения в терминах возможных миров:

(1a) Существует такой мир $w$, что все, являющееся красным в мире $w$, является также блестящим в мире $w$.

(1b) Для всего, что является красным, существует такой мир $w$, что оно в нем является также и блестящим.

(1c) Существует такой мир $w$, что все, являющееся красным в действительном мире, является блестящим в мире $w$.

В языке алетической модальной логики существует только два способа соединить фигурирующий в данном примере оператор возможности с квантором общности, который связывает предмет утверждения. Поэтому обычная (алетическая) модальная логика позволяет формализовать лишь (1a) и (1b):

(1)') $\diamond \forall x(P(x) \rightarrow Q(x))$

(1)') $\forall x(P(x) \rightarrow \diamond Q(x))$

В первом случае квантор находится в области действия модального оператора, во втором - наоборот. Перевод (1c) возможен, только если использовать оператор актуальности, который обеспечит референцию антецедента к реальному миру:

(1c') $\diamond \forall x(A P(x) \rightarrow Q(x))$

Для нас особенно важно, что дополнительные выразительные возможности, появляющиеся с введением оператора актуальности, напрямую касаются проблемы смешанных истин. Как показали Дэвис и Хамберстоун [9], имея произвольную обычную истину $p$, из нее можно легко получить два вида смешанных истин. Пусть $p$ - контингентное a posteriori, тогда

- $A p$ - необходимое a posteriori

- $p \leftrightarrow A p$ - контингентное a priori

Посмотрим теперь, как оператор $A$, тесно связанный с двумерной семантикой и концепцией смешанных истин, помогает раскрыть смысл ансельмова аргумента. 


\section{$5 \quad I Q M C N$ как модальный объект особого рода}

Одной из главнейших трудностей, связанных с анализом «единственного аргумента», является проблема интерпретации отношения «больше». Очевидно, что это «больше» должно схватывать интуитивное различие между интенциональным существованием объекта (в уме) и его реальным существованием (в действительности). В свете всего сказанного ранее, мне представляется естественным формализовать существование в действительности с помощью оператора $A$. $\mathrm{B}$ отличие от традиционных операторов $\square$ и $\diamond$, он тесно связан с нашей способностью к семантическому, а не фактическому сослаганию (ведь именно на этой способности основана перформативная интерпретация ансельмова доказательства).

Как было показано, формальные свойства оператора актуальности таковы, что с его помощью для любого контингентного апостериорного утверждения (причем не только истинного, но и ложного) можно подобрать его «смешанные» аналоги. В частности, для истинного $p$ априорным аналогом будет необходимо истинное $A p$, для ложного $p-$ необходимо ложное $A p$. Далее, для истинного $p$ необходимым аналогом будет априори истинное $p \leftrightarrow A p$, для ложного $p$ - априори ложное $p \leftrightarrow \neg A p$. Другими словами, сам оператор $A$ можно рассматривать как оператор, образующий априорный онтологически детерминированный (необходимо истинный или необходимо ложный) аналог произвольного предложения. Дуальный же оператор $C$, образующий необходимый эпистемически детерминированный (априори истинный или априори ложный) аналог произвольного предложения вводится следующим определением:

(C) $p=\left\{\begin{array}{l}p \leftrightarrow A p, \text { если } A p \\ p \leftrightarrow \neg A p, \text { если } \neg A p\end{array}\right.$

В свете этих фактов я предлагаю интерпретировать отношение «больше» в выражении «то, больше чего нельзя представить» как рефлексивное, несимметричное и транзитивное отношение между объектом, данным априори, и тем же самым объектом, данным апостериори.

(M) Объект, данный априорным образом, больше, чем необходимо тождественный ему объект, данный апостериорным образом.

Для надлежащей формализации этого принципа требуется первопорядковая модальная логика, и чтобы не вдаваться в технические детали, воспользуемся его более общей пропозициональной версией. 
(M') Эпистемически детерминированный аналог апостериорной пропозиции больше, чем сама эта пропозиция.

Построим предложение $g$, которое утверждает, что больше него ничего помыслить нельзя (т. е., что оно априори эквивалентно своему эпистемически детерминированному аналогу):

(G) $g \leftrightarrow a p(g \leftrightarrow C g)$

Если допустить, что $g$ ложно $(\neg g)$, то правая часть эквивалентности (G) тоже должна быть ложной: $\neg(g \leftrightarrow C g)$. С другой стороны, по определению (C), в случае ложности $g$ формула $C g$ должна иметь вид $g \leftrightarrow \neg A g$. Таким образом, мы имеем эквивалентность $\neg g \leftrightarrow \neg(g \leftrightarrow(g \leftrightarrow \neg A g))$. Сокращая данную формулу по законам классической пропозициональной логики мы получаем противоречие ( $g$ ложно, е. т. е. $g$ априори истинно). Следовательно, допущение было неверным, то есть $g$ на самом деле не может быть ложным.

Разумеется, приведенное выше рассуждение представляет собой несколько упрощенную и не вполне строгую версию ансельмова аргумента. Тем не менее, на мой взгляд, оно предает основную идею этого доказательства. Суть в том, что когда я говорю «iqmcn существует» и осознаю, что не владею точным понятием о iqmcn, мое утверждение, по сути, означает «[Во всех возможных мирах] Существует нечто, что я сейчас обозначил как $i q m c n$, но не исключено, что в других возможных мирах использованный мною термин „,iqmcn“ имеет совсем другой денотат (или не имеет его вообще)». При этом у меня нет никаких концептуальных средств, чтобы произвести кросс-идентификацию объекта, обозначенного мною как «iqmcn» (мне неизвестны его сущностные, необходимые свойства - свойства, которыми он обладает во всех мирах, в которых существует). С другой стороны, у меня нет никаких концептуальных средств, чтобы сконструировать класс предметов, которые хотя бы в каких-то мирах обозначаются термином «iqmcn»- свойство «именоваться iqmcn» релятивизировано к возможными мирам, имя «iqmcn» не является жестким десигнатором.

\section{Литература}

[1] Аверинцев С.С. Бытие как совершенство - красота как бытие // Аверинцев С.С. Поэтика ранневизантийской литературы. - М. : Coda, 1997. - C. 30-58. 
[2] Ансельм Кентерберийский. Прослогион // Ансельм Кентерберийский. Сочинения. - М. : Канон, 1995. - С. 123-165.

[3] Басос А.В. «Единственный аргумент» Ансельма Кентерберийского // Истина и благо: универсальное и сингулярное.-М.: ИФ PAH, 2002. - C. 146-166.

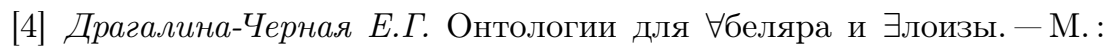
Изд. дом НИУ ВШЭ, 2012.

[5] Драгалина-Черная Е.Г. Via eminentiae как инференциальная и аргументативная стратегия // Полемическая культура и структура научного текста в Средние века и раннее Новое время. - М. : Изд. дом НИУ ВШЭ, 2012. - С. 243-258.

[6] Драгалина-Черная Е.Г. Тяжба о «ста талерах»: via eminentiae Кантовский сборник. - 2009. - № 2 (30). - С. 89-100.

[7] Крипке $C$. Тождество и необходимость // Новое в зарубежной лингвистике. - Вып. ХIII. Логика и лингвистика (Проблемы референции). - М. : Радуга, 1982. - С. 340-376.

[8] Chalmers D. The foundations of two-dimensional semantics // GarciaCarpintero M., Maciá J. Two-Dimensional Semantics.-Oxford: Clarendon Press, 2006. - P. 55-140.

[9] Davies M., Humberstone L. Two notions of necessity // Philosophical Studies. - 1980. — № 38. - P. 1-31.

[10] Kaplan D. Demonstratives // Themes from Kaplan.-Oxford: Oxford University Press, 1977. - P. 481-564

[11] Stalnaker $R$. Assertion revisited. On the interpretation of twodimensional modal semantics // Philosophical Studies. - 2004. Vol. 118. - P. 299-322.

Надійшла до редакиї 10 червня 2013 р. 